Cultures \& Conflits

12 | hiver 1993

L'action collective : terrains d'analyse

\title{
Barrer, filtrer, encombrer : les routiers et l'art de retenir ses semblables
}

\section{Guillaume Courty}

\section{(2) OpenEdition}

1 Journals

\section{Édition électronique}

URL : http://journals.openedition.org/conflits/445

DOI : $10.4000 /$ conflits.445

ISSN : $1777-5345$

Éditeur :

CCLS - Centre d'études sur les conflits lilberté et sécurité, L'Harmattan

Édition imprimée

Date de publication : 15 décembre 1993

ISSN : 1157-996X

Référence électronique

Guillaume Courty, «Barrer, filtrer, encombrer : les routiers et l'art de retenir ses semblables », Cultures \& Conflits [En ligne], 12 I hiver 1993, mis en ligne le 14 mars 2006, consulté le 30 mars 2021. URL : http:// journals.openedition.org/conflits/445; DOl : https://doi.org/10.4000/conflits.445

Ce document a été généré automatiquement le 30 mars 2021.

Creative Commons License 


\title{
Barrer, filtrer, encombrer : les routiers et l'art de retenir ses semblables
}

\author{
Guillaume Courty
}

1 Par trois fois ces huit dernières années ${ }^{1}$, les routiers ont barré les routes de France. En février 1984, novembre 1991 et juillet 1992. Ces mouvements qui étaient à l'origine localisés se sont diffusés sur pratiquement l'ensemble du réseau routier français (et même européen pour 1984). Ces mobilisations posent deux types de problèmes d'analyse. Le premier qui sera développé tout au long de cet article est celui de l'apparition et de la "réussite" de cette technologie particulière d'action collective consistant à barrer les routes. Le second tient dans l'hétérogénéité du groupe social : il n'est ni patronal, ni artisanal, ni salarial, ni ouvrier mais tout à la fois. Les routiers sont un groupe social qui fait croire en son existence et qui est le produit des différentes catégories sociales qui l'ont mobilisé à leur profit pour se redéfinir à partir du début des années trente ${ }^{2}$. On trouve alors un noyau dur constitué des entrepreneurs de différents métiers des transports sur route: déménageurs, loueurs de véhicules, commissionnaires, tractionnaires... On trouve également des conducteurs salariés de différents types de véhicules (les cars comme les différents types de poids lourds) transportant différents objets ${ }^{3}$. Si les routiers ont la réputation de barrer les routes, ces individus sont aussi bien des patrons que des conducteurs salariés ou artisans. Mais le fait marquant provient de ce que personne ne va chercher à distinguer l'entrepreneur du salarié : le fait de voir un camion entraver la circulation suffit à faire la preuve que "les routiers barrent les routes". Pourtant cette illusion ne résiste pas à l'analyse : les routiers ne barrent pas les routes, ils usent d'une technologie particulière d'action collective permettant de regrouper ses semblables, de manifester le groupe. Le groupe social des routiers constitue un terrain propice à la vérification de certaines hypothèses. Nous y avons notamment appliqué une analyse de "l'art de se regrouper". Cette notion vise principalement à reformuler les hypothèses de travail induites par la théorie des groupes d'intérêt. Elle s'articule à une sociogenèse de l'action collective et se donne pour objectif de permettre de repérer les individus qui usent et composent 
des formes qui, si elles ne sont pas le fruit de la folie ou de la maladresse, n'en sont pas moins imprévisibles dans les champs où elles sont l'objet d'investissements individuels, matériels et symboliques. Après plus d'un siècle de concurrence pour la représentation des routiers, la spécificité de leur art de se regrouper a abouti à la constitution de multiples groupements reconnus socialement comme non politiques dans un secteur d'activités économiques. Cet art repose sur plusieurs éléments constitutifs. Sur les compétences des différents individus constituant le groupe tout d'abord. On trouve alors l'art du placement: les entrepreneurs, par exemple, ne se regroupent durablement qu'à la condition de posséder un savoir-faire leur permettant de se déplacer socialement, de conforter leur position sociale en convertissant des capitaux valables dans leur entreprise (pour y être un "bon" entrepreneur) en capitaux monnayables dans les organisations représentatives (pour y être un "bon" membre ou un "bon" président). On trouve également pour les conducteurs, les différentes manières de se placer dans des lieux où ils se sentent autorisés à rencontrer d'autres semblables (garages, dépôts, restaurants), du temps libre que l'on peut partager, en bref, des formes primaires de regroupements sur lesquelles peuvent se superposer des formes de plus en plus objectivées jusqu'à rendre imperceptibles ces formes élémentaires de la vie économique. Cet art repose également sur la constitution d'un talent, celui des différents individus qui se présentent comme les porte-parole du groupe et tentent d'imposer, avec plus ou moins de succès, la croyance dans l'effectivité de leur travail de représentation. Cet art repose enfin, sur ce que Norbert Elias appelle l'art d'observer et de manier ses semblables. Il est le fait d'instance d'évaluation et d'officialisation des catégories légitimes de perception des formes de groupements investies: on trouve alors le ministère des Travaux publics et certains parlementaires qui interviennent pour imposer des formes de groupements (les confédérations patronales à partir de 1919), en censurer d'autres (les groupements considérés comme non conformes à l'ordre public), importer et enseigner certaines pratiques (les élections pour les délégués, le respect de la parole publique, les procédés rhétoriques) et, plus généralement, pour donner du crédit aux formes de groupements ainsi reconnues. Avec ces institutions apparaît, du fait du quasi monopole de la violence symbolique, un marché de l'art de se regrouper. Tenter de restituer la constitution de cet art dans un secteur économique est donc l'objectif premier de cette analyse. Un autre objectif tient dans la restitution et la compréhension de la constitution dans la France républicaine de ce que nous appelons le stock de formes d'organisations disponibles. Or cette entreprise est confrontée à deux problèmes d'analyse. Tout d'abord, à la confusion dans les études consacrées à la mobilisation des groupes sociaux de deux dimensions de la sociogenèse de cet art : le "travail de regroupement" dans des organisations formelles de représentation d'un intérêt économique commun dans le travail de définition et de délimitation de cette catégorie ${ }^{4}$. Situer ces deux dimensions dans la même période - celle des années trente pour les cadres étudiés par Luc Boltanski - revient en fait à ne travailler ces individus qu'en situation sans comprendre leurs dispositions sociales et cet art de se regrouper qui est déjà là. En second lieu, cette entreprise est confrontée aux réponses ordinaires qui sont habituellement formulées pour expliquer pourquoi des individus se regroupent. Il est ainsi communément admis qu'on se regroupe grâce à des formes juridiques fournies clés en main ou encore du fait même que l'on partage le même intérêt économique (équivalent ici du métier). Mais quand le droit ou l'économie ne suffisent plus à eux seuls à rendre compte des regroupements présents, intervient ce qu'il est convenu d'appeler le spontanéisme. Ce 
penchant pour le spontanéisme se retrouve souvent et notamment lors de l'interprétation des conflits sociaux, périodes pendant lesquelles des individus se regroupent, mais sans avoir recours aux formes juridiques de regroupements. Un barrage routier, une coordination ou encore une délégation ne peuvent s'expliquer par le texte juridique qui les institue. Comment expliquer ces actions collectives si ce n'est en ayant recours à des travers psychologiques communs aux individus (un "naturel revendicatif") ou, le plus souvent, au caractère spontané de l'événement? Dire des routiers qu'ils sont naturellement enclins à la révolte revient à ne rien dire du tout si ce n'est à reprendre la stéréotypée du groupe à son propre compte. Faire dire aux routiers que leur action repose sur le ras le bol et le dégoût de la politique revient en effet à croire dans la stéréotypée de ce groupe. Les différentes techniques consistant à barrer, occuper, filtrer et encombrer les routes sont utilisées depuis longtemps sans pour autant avoir été l'apanage des seuls routiers: les Ponts et chaussées et plus généralement la force publique ont ainsi instauré les barrières pour contrôler les véhicules. En dehors des agents de l'État, les agriculteurs y ont également recours. Les conducteurs et entrepreneurs, eux, n'ont pas immédiatement utilisé cette technologie ou bien ne l'ont pas immédiatement dénommée un "barrage". En revanche, en s'en saisissant, ils ont transformé cette technologie d'action collective et l'ont différenciée en autant d'usages qu'il y a de catégories sociales distinctes dans ce groupe social. Dans la même période, ils ont abandonné différentes techniques d'encombrement des rues et de guérilla urbaine. Cette technologie semble de prime abord très simple à mettre en œuvre. Après tout, quoi de plus facile que de barrer une route lorsque l'on possède un véhicule qui en fait à lui seul plus de la largeur. Il suffit simplement de "se mettre en travers" et de couper le moteur. Si cette action est si simple, pourquoi n'est-elle pas plus souvent entreprise? Pourquoi n'a-t-elle pas été utilisée dès l'apparition des premiers poids lourds? Les barrages routiers passent de l'expression symbolique d'une maxime symbolisant l'état des représentants des syndicats professionnels à une technologie d'action collective consistant à regrouper ses semblables sans toujours leur demander leur consentement. Dans les années trente, les porte-parole des groupements utilisant concurremment le catégorème routier se servent de la métaphore du barrage pour exprimer la situation dans laquelle ils se trouvent. Cette expression se trouve à la FNMT-CGT qui se demande "quels moyens peut-on employer pour barrer la route aux prétentions des banques et des transporteurs qui sont seulement contrôlés par les banques"5. Elle se trouve à la Fédération nationale des transports routiers (groupement patronal majoritaire) où un des membres dirigeants constate "qu'on cherche à barrer la route aux transports routiers par tous les moyens"6. Cette métaphore est plus qu'une simple image utilisée pour rendre la réalité plus sensible aux membres des syndicats. Elle symbolise autant l'absence de prise en compte des intérêts de la profession par les pouvoirs publics que les pratiques policières qui tendent souvent à arrêter et immobiliser les véhicules poids lourds et ce depuis la création de la police de la route en 1929. Cette métaphore provient également des expériences intériorisées de barrières routières instituées par la puissance publique (des ponts à bascule de l'administration aux octrois des villes). Tous les individus du transport quelque soit leur activité et leur véhicule ont connu et connaissent les différents types de barrières administratives parce que ce sont elles qui leur barrent la route dans certaines situations. Ce sont également d'elles que partent les manifestations de conducteurs du début du XXe siècle.

Archéologie d'une technologie d'action collective 
2 Cette forme d'action collective n'est ni unique ni simple à mettre en œuvre et les différentes formes de barrages routiers qui la composent constituent une de ces "médiations concrètes"7 par lesquelles des individus se repèrent dans l'espace social et peuvent ainsi regrouper leurs semblables sur l'espace routier. Il y a différents moyens mobilisés pour barrer une route qui correspondent aux différents individus qui les utilisent et, donc aux différents usages de cette action collective. L'histoire de cette technologie peut se résumer de la sorte: d'origine urbaine elles sont sorties de cet espace sans possibilité de retour. Liées aux groupements confédérés de chauffeurs, elles deviennent pour les différents types de routiers un moyen de faire grève sans faire la grève $^{8}$. Pour le sociologue, ces conjonctures permettent d'observer une régression vers les habitus ${ }^{9}$, c'est-à-dire un ajustement des dispositions intériorisées avec les conditions de la situation ${ }^{10}$. Les barrages routiers reposent sur les mêmes formes élémentaires de vie collective que nous avons rencontrées pour l'art de se regrouper dans des groupements dits formels.

De la rue à la route

3 Historiquement cette manière de manifester n'apparaît pas comme particulièrement spécifique aux routiers car l'interruption de la circulation n'est pas l'apanage des seuls personnels de conduite. En revanche, l'interruption volontaire de la circulation et le repérage des véhicules poids lourds deviennent une technologie particulière d'action collective qui se dégage dans les groupements confédérés et se diffuse dans l'ensemble du champ de représentation des routiers. Le début du XXe siècle est marqué par l'apparition de manifestations urbaines des personnels de conduite dans des lieux où les conducteurs sont déjà arrêtés ou attendent de reprendre la route (garages, dépôts, octrois, douanes...). Les différents conducteurs confédérés maniaient parfaitement la technologie de l'encombrement urbain. "Il y a l'embouteillage qui consiste à encombrer la voie publique de façon à rendre impossible toute circulation ${ }^{11}$ Puis le boycottage qui consiste à violer les nouveaux règlements de la circulation et à passer outre aux injonctions des agents"12. Mais, pendant de nombreuses années, l'action tendant à barrer la route aux autres usagers n'est pas utilisée. Les conducteurs se cantonnent dans les rues et utilisent l'embouteillage urbain là où circulent le plus de véhicules et d'usagers différents de véhicules. Tout se passe comme si ces conducteurs, issus en grande partie des milieux ruraux, une fois implantés dans les centres urbains, voulaient y démontrer symboliquement leur présence. On note alors les conflits menés par les chauffeurs de taxis et racontés par L. Aragon dans Les cloches de Bâle. Après la première guerre mondiale les camionneurs agissent souvent. En 1933, la conjonction des taxis parisiens, des voyageurs de commerce et des poids lourds aboutit à "une manifestation dans les grandes artères parisiennes, embouteillant la circulation"13. Une autre forme de démonstration apparaît et vient s'ajouter à celle de l'embouteillage volontaire. C'est lors des meetings, et plus exactement après les meetings, que des défilés et cortèges se développent chez les conducteurs salariés et dans Paris. En 1909, une "manifestation d'environ 200 voitures automobiles ont circulé à la file et en bon ordre à travers les rues de la ville"14. Aux défilés et cortèges s'ajoutent les stratégies de prise symbolique de l'espace urbain. Mais ce n'est pas tant la rue qui est visée que ceux qui l'utilisent. En 1910, lors d'un meeting de chauffeurs apparaît "une manifestation originale. Les chauffeurs d'automobiles, réunis hier au soir en meeting ${ }^{15}$ ont décidé de procéder en guise de protestation, à une manifestation qui aura lieu un de ces jours et qui consistera à se réunir en tel nombre, sur un point précis et très fréquenté de la capitale, que pendant une heure ou deux toute espèce de circulation sera impossible"16. 
Peu de lieux parisiens répondent aux critères édictés ici. Pour les chauffeurs, il n'en existe qu'un : les barrières d'octroi. Elles retiennent déjà la circulation, sont forcément fréquentées et permettent de fermer Paris à la circulation. C'est à partir de ces barrières qu'en juin 1911 des manifestations s'organisent pour lesquelles les leaders commandent de se "rendre sur les grands boulevards ${ }^{17}$ pour interrompre la circulation"18. C'est donc sur les lieux où la rue est déjà barrée que les chauffeurs se retrouvent pour commencer à manifester. Là, nous entrevoyons la dimension stratégique de toutes ces techniques d'encombrement urbain. Ce n'est pas la circulation en tant que telle qui est visée mais certains véhicules qu'il faut trouver pour s'en accaparer. Trouver les barrières existantes et s'en emparer devient le préalable à l'action collective: ce n'est qu'à partir de ces barrières que peuvent partir les manifestations car ce n'est que dans ces lieux que les agents trouvent ceux avec qui ils veulent manifester, c'est-à-dire les autres chauffeurs. Ces formes d'action collective reposent donc en fait sur l'art de se regrouper qui permet de composer un collectif grâce à un collectif déjà existant mais sur un autre mode. Les barrages routiers sont pour nous l'occasion de voir se manifester le "noyau dur" des routiers c'est-à-dire l'ensemble des agents qui ont mobilisé ce terme pour leur propre usage. C'est également l'occasion de retrouver les autres conducteurs de véhicules à usage professionnel qui ont peu à peu renoncé à barrer les routes pour ne laisser que les routiers utiliser cette technologie. Si l'embouteillage ne vise pas à interrompre la circulation mais à retrouver ses semblables pour mieux manifester, cette technique n'est pas toujours très efficace. L'encombrement et l'embouteillage se doivent de permettre le rassemblement mais également la mise à l'écart des éléments avec lesquels on ne veut pas interagir et être vus. Nous retrouvons ici aussi un enjeu de l'art de se regrouper : il faut certes associer des semblables mais également dissocier les autres et se mettre à l'abri des forces de police. Dans les rues, des techniques particulières sont utilisées pour arrêter les véhicules industriels récalcitrants, faire sortir de la nasse ceux qui ne doivent pas y figurer durablement et éviter les contacts avec les forces de l'ordre. Les attaques de véhicules et les piquets roulants sont ainsi utilisés pour capturer des véhicules industriels qui continuent à circuler malgré les mots d'ordre d'arrêt de la circulation. Dans les années trente, Paris et les grandes villes restent le centre d'intérêt des routiers mais ce n'est plus le centre urbain qui les intéresse : le centre politique est visé. En 1933, "les transporteurs descendent dans la rue ${ }^{19} l^{1}$ embouteillent ${ }^{20}$ mobilisant la Police et livrent à leur façon un premier assaut contre la Chambre des députés" ${ }^{21}$. Si le politique devient la cible de ces manifestations, les autorités administratives sont également au centre de ces dispositifs. Le 14 février 1947 "dans toutes les préfectures et sous préfectures de France, un cortège impressionnant de véhicules des transporteurs publics a montré à tous que les routiers n'étaient pas cette fois décidés à se laisser faire" ${ }^{22}$. C'est dans ces années trente que la technique du barrage passe d'une expression symbolique à une action collective. Elle est alors utilisée par pratiquement tous les entrepreneurs de transports et les conducteurs de véhicules à usage professionnel. C'est dans les années soixante que les routiers se transportent progressivement en dehors des villes. C'est en 1956 que la technologie du barrage est utilisée systématiquement sur les axes routiers même si les centres ville sont encore le cadre d'embouteillage volontaire.

L'exode urbain

4 L'exode urbain a lieu à la fin des années cinquante. A la Libération, les transporteurs et les conducteurs tentaient toujours d'occuper le pavé parisien. Mais ces manifestations 
n'avaient rien de spontané. Elles étaient au contraire coordonnées avec d'autres mobilisations. Entre 1946 et 1947 de nombreux meetings et salons professionnels se déroulent. Ils sont assortis de différents défilés de véhicules dans la capitale. On assiste alors à une division des tâches : pendant que les uns prennent la parole dans les salles et rencontrent les autorités, les autres roulent dans les villes. On assiste également à la diffusion du savoir-faire des groupements confédérés dans les groupements patronaux mais mis en œuvre en dehors de villes cette fois. Après ces années, les camions n'ont plus manifesté dans Paris. Quelques rares incursions ont néanmoins eu lieu mais sans véhicules. Paris n'en reste pas moins au centre des débats et lorsque les routes sont bloquées, la presse professionnelle peut affirmer que "Paris n'est pas bloqué" ${ }^{23}$. Les forces de police, elles, prennent toutes les mesures pour empêcher les véhicules de rentrer dans la capitale dans les périodes de mobilisation ${ }^{24}$. Comment expliquer ce renoncement à agir dans les villes? Trois processus se sont surajoutés les uns aux autres. Le premier est la sortie des centres urbains des locaux des entreprises de transports et des sièges sociaux de certains groupements professionnels: les technologies auraient ainsi suivi les hommes et leur intériorisation d'un nouvel élément de leur façonnement psychologique. Un deuxième élément tient dans les transactions établies avec les pouvoirs publics qui permettent ces manifestations mais en dehors des centres villes. Le troisième et dernier tient dans l'hypothèse que nous avons formulée concernant les barrages : s'ils constituent des technologies permettant de repérer et regrouper ses semblables, les villes sont délaissées car ceux avec qui ces individus souhaitent faire corps n'y circulent plus ni en quantité suffisante, ni en qualité professionnelle. Les "chauffeurs-livreurs" qui circulent en ville ne possèdent pratiquement aucun des attributs à même de les faire prendre pour des routiers : ni le véhicule, ni l'héxis, ni la route. Sorties des villes, ces manifestations prennent un nom et un seul, les barrages. Elles sont utilisées par et pour une seule catégorie : le noyau dur des routiers.

Un rituel hivernal

Dès le début des années soixante les barrages sont routiers. A peine utilisés par ces agents, les barrages perdent de leur signification syndicale pour être expliqués le plus souvent par des raisons climatiques ou psychologiques. Les routiers barrent les routes, c'est un fait acquis, mais ils ne le font que lorsqu'ils sont déjà eux mêmes bloqués par les conditions climatiques (barrières de dégel, circulation interrompue par la neige...) ou lorsqu'ils manifestent spontanément leur "ras le bol" contre une nouvelle mesure gouvernementale (une hausse des carburants ou une réforme du permis de conduire). Cette apparition de la causalité climatique ou psychologique constitue un des indices de la réussite de cette technologie d'action collective dans ce champ de représentation. $\mathrm{Si}$ les groupements professionnels en sont les instigateurs au début des années soixante, les barrages dépassent rapidement leurs capacités une fois qu'ils prennent la dimension nouvelle d'événement politique. Il s'ensuit des stratégies de démarquage de certains groupements d'entrepreneurs comme de salariés vis-à-vis de ces événements et des représentations qui sont alors produites sur les routiers. La plupart du temps, les porteparole patronaux eux-mêmes ont recours aux explications de ces événements qui font état des conditions climatiques ou des caractéristiques psychologiques des seuls conducteurs. Chaque porte-parole essaye ainsi de dédouaner son organisation de toute responsabilité directe en utilisant ces catégories pratiques que sont devenus les routiers et leur mythologie. Pour les agents de ce champ, ces actions collectives ont donc leur propre cause. La régularité des conditions climatiques dans certains lieux 
stratégiques en est une. Une autre tient dans la mise en évidence d'un modèle stimulus / réponses : les routiers sont censés avoir des propensions naturelles à barrer les routes en répondant à des stimuli précis ${ }^{25}$. Cette technologie semble même résulter peu à peu d'un rituel quasi annuel qui se déroule chaque hiver lorsque des conducteurs sont bloqués aux frontières soit du fait des conditions climatiques, soit comme en 1984 du fait des mouvements de grève des douaniers aux postes frontières. Mais quelle que soit la saison ou le lieu, l'ensemble des groupements du champ s'empare de cette technologie dans les années soixante-dix. La presse politique nationale exprime sa désapprobation de plus en plus nettement, estimant que cette situation doit cesser. Elle s'empare de ces "routiers qui bloquent"26. Ils bloquent les routes, certes, mais surtout les vacanciers et les automobilistes qui n'y sont pour rien, quand ils ne vont pas jusqu'à bloquer le ministre des Transports lui-même ${ }^{27}$.

"La France bloquée"

6 Les mois de février 1984 et juillet 1992 sont des conjonctures permettant la vérification des hypothèses précédemment émises sur la sociogenèse de cette technique d'action collective. Ces deux conflits routiers nous permettent d'affiner cette première conception des barrages comme entreprise de repérage et processus de sélection sur l'espace routier de ceux qui doivent être groupés avec vous, autour de vous. Dans les années quatre-vingt, les barrages offrent en plus aux agents un moyen de prendre symboliquement la ville en la bloquant, de distinguer les habitants des villes de ceux de la campagne, les usagers de la route des professionnels de la route.

1984

7 L'un des problèmes insolubles de ces événements est de savoir quand les barrages ont commencé. Dans la première quinzaine de février, les douaniers italiens font grève deux jours par semaine après que les transporteurs italiens aient eux-mêmes fait grève. Le 14, les douaniers français prennent le relais. De nombreux routiers étaient déjà bloqués aux frontières depuis plusieurs jours et ont décidé de bloquer à leur tour les lieux sur lesquels ils sont retenus. L'entrée dans cette action était donc d'un coût quasiment nul car on ne perd rien à barrer un lieu sur lequel on est soi-même immobilisé. Jusque là nous sommes dans un schéma classique : les postes frontières sont des lieux dans lesquels les conducteurs attendent et ce faisant se regroupent selon certaines logiques. Il n'y a ni mot d'ordre de blocage, ni même arrêt de son activité pour manifester son mécontentement, il y a simplement une occupation des lieux sur lesquels on est retenu et sur lesquels on a l'habitude d'attendre. Cette situation s'étend mais en restant toutefois cantonnée à la région de la Haute-Savoie jusqu'au samedi 18 février. A partir de ce jour, les barrages se développent dans certaines régions et à des endroits bien précis : Albi, Compiègne, Reims, Nancy, Strasbourg, Clermont-Ferrand. Au fur et à mesure, les barrages s'étendent autour de ces lieux et forment des zones de barrages: Normandie, Bretagne, Picardie, Alsace, Midi Pyrénées, Provence, Centre, Savoie et Saône. Ce processus s'accélère entre le mardi 21 et le mercredi 22. On passe ainsi en deux jours de 21 à 36 départements touchés et de 48 à 155 barrages. Au delà des chiffres mêmes, cette dissémination est à plus d'un titre impressionnante. Le Centre national d'information routière cesse de faire une évaluation des barrages à partir du mercredi 22 car il ne dispose plus de moyens techniques suffisants et, surtout, ne sait plus quoi recenser. Les villes sont aussi l'objet d'une occupation spécifique : Reims et Clermont-Ferrand sont barrées aux quatre points cardinaux. D'autres lieux sont privilégiés: les échangeurs d'autoroutes, des routes nationales et quelques chemins départementaux. De plus, la majorité des barrages sont filtrants et retiennent plus 
particulièrement les véhicules de transports. $\mathrm{Si}$, ni le froid, ni la chaleur ou les douaniers ne sont directement et uniquement en cause comment peut-on comprendre une telle diffusion de la mobilisation? Au départ, des postes frontières sont concernés or seule une catégorie de transporteurs et conducteurs passent par les postes frontières, il s'agit des internationaux. Le problème devient alors le suivant : pourquoi la mobilisation s'est-elle propagée à des agents "étrangers" aux premiers manifestants c'est-à-dire n'ayant ni les mêmes lieux de travail, ni les mêmes impératifs professionnels, ni les mêmes intérêts économiques que les internationaux, les "seigneurs de la route" ? Il existe une multiplicité de moyens de barrer une route même si la finalité peut sembler la même: les agents opposent souvent les opérations "escargots" aux barrages fixes ou filtrants. Tout d'abord un barrage consiste en un point stratégique qui n'est pas choisi au hasard. De même que l'on ne défile pas devant n'importe qui ni n'importe où, on ne barre pas n'importe quelle route. Il faut donc choisir consciemment ou inconsciemment le point crucial et l'investir. Ensuite, il faut le tenir. Un barrage peut être constitué d'un seul camion comme de mille et dans ce cas nous ne pouvons pas savoir quelle est la proportion de conducteurs barrant la route et celle de ceux qui y sont barrés ${ }^{28}$ pour s'extraire de la nasse où ils étaient retenus en otage". Cf. Libération, 7 novembre 1991.]]. Le choix de telle ou telle technique de barrage n'est pas le fruit du hasard. Ce choix repose principalement sur les catégories sociales qui les utilisent. Il nous faut donc distinguer les techniques destinées à empêcher les "civils" de passer et celles visant principalement les routiers.

Des véhicules de choix

8 Si les barrages permettent de repérer ses semblables pour les grouper ensemble, les agents se servent d'un objet pour effectuer ce repérage : il s'agit du poids lourd. Le véhicule retrouve sa dimension de marqueur social objectivée ${ }^{29}$ qui permet de savoir à tout coup à qui on a à faire. Mais ce n'est pas seulement le type du véhicule qui compte, les objets qui y sont attachés sont également déterminants. Le nom de l'entreprise qui y figure, les plaques d'identification de la marchandise, sa spécialisation (citerne, frigorifique, porte voitures...), et même les objets mis en évidence dans la cabine du conducteur permettent de repérer à travers les véhicules les individus intéressants et, à partir de là, de manifester un groupe. Certains véhicules sont aujourd'hui délaissés lors de ces mobilisations. C'est le cas principalement pour les cars alors même qu'ils sont liés historiquement au secteur des transports routiers et que leurs entrepreneurs et conducteurs ont connu la même trajectoire historique et partagent parfois encore les mêmes groupements professionnels. En effet, ceux que l'on se met à dénommer "routiers" dans les années trente sont aussi bien camionneurs que conducteurs de cars mais les véhicules de transport en commun ne constituent plus des objets retenus pour manifester le groupe des routiers : les cars ne sont pas des véhicules que l'on retient dans les barrages alors que les omnibus étaient des véhicules recherchés dans les centres urbains et faisaient souvent l'objet d'attaque et de défilés victorieux une fois le véhicule capturé. Aujourd'hui, on se contente de les retarder et non pas de les arrêter pour retenir le chauffeur avec soi. Nous ne signifions pas par là qu'aucun car n'a été arrêté ou retardé en chemin par un barrage, nous signifions que les cars ne sont plus perçus comme un véhicule de routier. La même règle s'applique pour les véhicules de transport que tout titulaire d'un permis tourisme peut conduire: les petits camions, camionnettes et autres fourgonnettes, eux aussi ne sont pas retenus. Il en va de même pour les autres professionnels de la route qui, auparavant, embouteillaient la capitale avec les camionneurs. Les routiers soulignent qu'ils laissent passer les ambulances, les 
VRP et les taxis. En dehors des professionnels de la route, les porte-parole des barrages les moins étanches tiennent à préciser qu'ils laissent passer les véhicules "à bord desquels se trouvent des enfants" ${ }^{30}$ ou un autre parce que la conductrice a de "jolis yeux". Lorsque le "bon" véhicule est repéré comment l'arrête-t-on? Le plus souvent, le conducteur s'arrête de lui-même pour aller voir les autres et puis s'en va prévenir son employeur qu'il est bloqué. Lorsqu'un conducteur ne veut pas s'immobiliser, nombre de moyens sont disponibles: de la persuasion la plus douce aux procédés plus énergiques exercés sur la personne du conducteur ou sur son véhicule (pneus crevés ou dégonflés). Certains trouvent des arrangements avec les initiateurs du barrage et obtiennent la permission de partir avec le seul tracteur mais en échange de leur chargement qu'ils doivent laisser sur place ou, quoique plus rarement, avec la remorque mais en promettant qu'au moins rien ne sera livré dans la ville. Pour trouver ces véhicules, il faut trouver les axes routiers les plus fréquentés et, si possible, les points de passage obligés : unique pont sur un fleuve, bretelles d'accès à une autoroute, axe obligatoire pour poids lourds, voie d'accès aux postes de dédouanement ou aux bureaux du transitaire, parkings, zones de fret, restaurants très fréquentés... Ces lieux permettent de trouver un grand nombre de poids lourds déjà présélectionnés et également de disposer de lieux de sociabilité qui sont organisés pour les conducteurs. Cette sélection joue surtout à partir du moment où les barrages ne sont plus uniquement des lieux de manifestations, mais des lieux de vie sociale. Il est à cet effet important de noter que certains barrages se transforment en lieu permanent de manifestations c'est-à-dire que, contrairement à certains autres qui sont installés le matin pour être levés le soir, des barrages sont des lieux où il faut vivre. Ainsi se forment des groupes structurés où le travail est divisé : ils élisent un responsable et un adjoint, seules personnes habilitées à discuter avec les autorités de police ou préfectorales. D'autres s'occupent de trouver des fonds pour le ravitaillement en cigarettes, boissons, nourriture. A chaque fois qu'un problème se pose, le responsable se déplace et tente de régler celui-ci quelle que soit sa nature. Dans ces groupes dotés de porte-parole réapparaissent les formes élémentaires de vie collective. Il leur faut maintenant attendre, écouter la radio pour savoir ce qu'on dit d'eux, regarder l'intervention télévisée du président d'un syndicat ou du ministre, parler au nom des autres, boire et manger ensemble. Ces barrages même s'ils sont différents reposent sur les mêmes catégories de perception et les mêmes dispositions intériorisées qui objectivent une autre forme d'action collective dans certaines conjonctures. Au semblant de désordre et de violence "naturelle" que les barrages procurent, il faut au contraire saisir l'ordre qu'ils créent. Si les barrages permettent de se focaliser sur certains véhicules et de constituer des groupes d'individus assez homogènes, les différentes technologies se répartissent selon les différents individus qui les utilisent et en fonction de ceux qu'ils veulent repérer et regrouper.

La dispersion sociologique

9 Nous avons effectué un découpage des barrages recensés par le CNIR ou évoqués dans les dépêches d'agences selon les régions touchées afin de voir quels étaient les processus de mobilisation. L'objet de ce découpage est de trouver les logiques d'utilisation de telle ou telle technologie d'action collective. Il apparaît alors nettement que ce ne sont pas "les plus faibles ${ }^{31}$ se montrent les plus agressifs lorsque la situation paraît avantageuse" ${ }^{32}$ mais les techniques utilisées qui sont plus ou moins contraignantes eu égard à l'objectif visé : forcer les autres à s'arrêter pour faire corps avec soi. Selon les groupes en présence, les modalités de l'action, les mobiles invoqués et les lieux choisis ne sont pas les mêmes. Les "barrages" des syndicats patronaux sont 
le plus souvent filtrants. Au contraire, les barrages "spontanés" des salariés et artisans sont fixes. Si les barrages sont une technologie d'action collective permettant de regrouper derrière soi ses semblables, les entrepreneurs et les salariés n'utilisent pas les mêmes technologies parce que les semblables ne sont pas les mêmes. Les conducteurs retiennent les leurs en se focalisant sur l'arrêt complet du plus grand nombre de véhicules là où les entrepreneurs et artisans filtrent les poids lourds jusqu'à trouver les véhicules des entreprises qui les intéressent (les concurrents, étrangers, "mangeurs de boulots"...). En tout cas ils tentent de marquer les automobilistes en leur expliquant leur action et en leur faisant pour une fois perdre leur temps. Si les véhicules sont l'objet d'autant d'attention, les personnes physiques peuvent néanmoins passer entre les différents barrages. Des barrages deviennent des lieux vers lesquels convergent certains individus. Les différents journalistes, mais également les élus locaux, représentants des forces de l'ordre, hauts fonctionnaires organisant les négociations et représentants de groupements professionnels. Les barrages sont des instruments de distinction mais ils sont également des groupements peu formels desquels émergent des revendications et des leaders.

L'auteur du "vrai cahier" de revendications

Ces barrages créent une hiérarchie en plus de la division du travail déjà envisagée. Des acteurs sont promus représentants d'un barrage et deviennent les porte-parole des leurs. Par trois fois, les barrages routiers ont permis l'émergence de ce que les journalistes appellent un "leader des inorganisés". En juillet 1992, et ce pour la première fois, ce leader est un conducteur (Tarzan) alors qu'en 1984 c'était un artisan et en 1991 un entrepreneur. En 1984, le leader "inorganisé" qui émerge est l'auteur d'un cahier de revendications grâce auquel les négociations peuvent commencer. Cet individu (M. Vasseur) n'est pas le seul à avoir mis par écrit les revendications des siens mais il est le seul à occuper le devant de la scène pendant tous ces événements. En 1991, le porte-parole consacré par les pouvoirs publics et la presse est un ancien candidat à la présidence de la FNTR en rupture avec son groupement et en passe de créer la coordination des transports routiers. En 1992, Tarzan est le routier plus vrai que nature (plus vrai que nature), l'exemple typique conforme à la stéréotypée du routier. Il est également celui auquel les médias, les pouvoirs publics et certains individus sur les barrages reconnaissent la capacité de parler et de représenter cette catégorie. Il est surtout celui grâce à qui les pouvoirs publics pensent pouvoir ramener les autres à la raison en leur montrant qu'ils sont écoutés et même reçus par le Premier ministre. L'apparition de ces leaders est un des effets émergents de ces conjonctures ${ }^{33}$. Avec eux, l'espace social des routiers devient ternaire. Celui-ci se concevait jusqu'alors par l'opposition des groupements patronaux aux groupements de salariés. Il existe désormais une troisième voie celle de cet individu qui prend la parole en dehors de toute organisation. Ensuite c'est grâce à eux qu'apparaissent les revendications des routiers. Sans revendication, il n'y a pas d'évaluation possible. Personne ne peut définir une "zone contractuelle" c'est-à-dire un terrain sur lequel s'entendre. Or ce sont ces revendications qui sont retenues pour faire l'objet des négociations et permettent d'entrevoir l'issue du conflit. Comme tout effet émergent, leurs positions ne sont pas stables. Leur personne même est mise en cause soit physiquement, soit verbalement car peu de représentants d'intérêts en place désirent l'intégration d'un tel joueur ${ }^{34}$. Les autres leaders des barrages n'admettent pas non plus qu'un des leurs fasse l'objet de tant d'attention.

Les vérifications de l'extension du mouvement 
11 L'enjeu des barrages se transforme avec l'intervention de l'armée. En 1984, la force armée participe à l'extension et à la radicalisation du mouvement. En 1992, l'envoi des chars contre les camions a arrêté la mobilisation. Avec l'envoi de la troupe, les individus présents sur les barrages s'attachent à vérifier que le plus grand nombre d'individus entrent dans le conflit et ce quels que soient leurs véhicules, les entreprises pour lesquelles ils travaillent ou leur statut juridique. En 1984, un mot apparaît, celui de solidarité. Il n'est ni une façade ni un prétexte mais le moyen de "vérifier que d'autres unités naturelles de l'espace de mobilisation entrent également en mouvement ou sont sur le point de le faire" ${ }^{35}$. Certaines routes changent de dimension stratégiques. Jusque là, elles étaient barrées en plusieurs endroits par différentes catégories de conducteurs et d'entrepreneurs. L'arrivée des chars et des grues unifie ces barrages et ceux qui les animent: ils ne font plus qu'un contre les autres. L'entrée dans le jeu de l'armée influe également sur les individus qui sont "chez eux", dans leur région, et qui oublient momentanément les revendications ou inimitiés professionnelles pour défendre leur sol (ce phénomène a surtout été sensible dans les Alpes en 1984). Dans d'autres départements, nombre de barrages sont mis en place et participent à la dispersion du mouvement, chacun ayant sa manière d'agir et ses revendications. L'armée a certes aidé à l'extension de la mobilisation, mais la diffusion des barrages a, elle, aidé au brouillage des éléments de compréhension de ce qui se passe. Le mardi 21 février 1984 au soir, peu d'individus sont encore à même de comprendre qui agit, pourquoi il agit et ce qu'il revendique. En juillet 1992 au contraire, deux faits participent à la levée des barrages. Le premier est constitué par l'épreuve de force directe contre les camions et non contre les hommes. Les pouvoirs publics ont ainsi atteint symboliquement cette catégorie sociale qui fait "corps avec son véhicule" et incité à la démobilisation. Le second est constitué par le mythe des routiers morts qui se propage à partir de Lyon suite aux échauffourées entre forces de l'ordre et membres des barrages dans le quartier de Gerland. Ce mythe clôt la mobilisation en assurant les conducteurs que la population "a pris fait et cause pour eux"36 et que ceux qui ont dépassé la mesure se sont désormais les autres, les forces de l'ordre.

Les techniques de résolution du conflit

Résoudre un conflit de cette dimension devient un enjeu important pour le ministre des Transports et ses collaborateurs et ce encore plus lorsque d'autres autorités gouvernementales entrent en jeu. Pour Pierre Mauroy, Premier ministre en 1984, les routiers français représentent le mythe du Chili. Il décide d'étouffer immédiatement un tel mouvement en envoyant l'armée car "c'est un problème d'autorité, il faut envoyer la troupe"37. Le premier coup envisagé par le Premier ministre est donc le recours à l'armée, à la médiation - faire jouer un coup par une instance autre que la vôtre. L'efficacité d'un tel coup est souvent peu réelle du fait d'interférences entre la prise de décision et son exécution. En l'espèce, les décisions prises subissent des traductions en chaîne à chaque fois qu'elles passent par une agence d'exécution différente. Cette réaction du Premier ministre traduite par l'équipe du ministre des Transports (Charles Fiterman) devient "l'idée d'envoyer la troupe pour enlever les camions. Mais je crois qu'il ne devait y avoir que quelques grues dans toute la France susceptibles d'enlever ceux-ci. C'est à ce genre de détails que l'on s'aperçoit que certains types de conflits n'ont jamais été planifiés et où la réaction de la police, gendarmerie, troupe est inadéquate" ${ }^{138}$. En passant successivement du Premier ministre au ministre des Transports, l'envoi de la troupe se transforme en grues pour déplacer les camions. Sur les barrages cela permet aux patrons transporteurs de réquisitionner les conducteurs 
de ces grues, qui, bien que conducteurs de travaux publics, n'en sont pas moins des conducteurs. Cette même phrase de Pierre Mauroy interprétée maintenant par certains préfets donne d'autres traductions. Certains envoient en effet la troupe et les engins motorisés. Nous ne savons pas comment ces engins sont entrés en action, en revanche nous avons recueilli la répercussion d'une telle arrivée sur la dimension de certains barrages. Certains y perdent leur caractère "bon enfant" pour devenir des places fortes difficilement prenables d'assaut - à Cluses certains vont jusqu'à miner les camions en tête de barrage. D'autres coups sont joués et reprennent les stigmates des routiers. Les routiers, dont on souligne volontiers le franc-parler, font eux-mêmes leur radio grâce à la CB : l'armée les réduit au silence en brouillant les fréquences pour empêcher des informations stratégiques de passer entre conducteurs. Les routiers ne possèdent comme preuve juridique de leur statut social que leur permis de conduire et leur respect des règles de conduite. Les camions qui barrent les routes sont considérés comme une entrave à la circulation et font l'objet d'une contravention quand les gendarmes ne dressent pas directement des procès verbaux d'infraction et procèdent aux retraits de licence. Pourtant en 1984, le ministre des Transports avait demandé par directive qu'aucune mesure coercitive ne soit prise à l'encontre des routiers sur les barrages: le ministre de la Défense affirme quant à lui ne pas avoir reçu une telle directive. En revanche, l'activité des ministères est parfaitement coordonnée en matière budgétaire lors de la parution au Journal officiel de l'arrêté concernant l'augmentation générale des tarifs de transports routiers de marchandises. La même synergie caractérise la mise en liquidité des 800000 francs nécessaires à l'indemnisation des routiers immobilisés dans la Maurienne - 223 d'entre eux sont ainsi indemnisés. En 1984, l'entrée en jeu des professionnels de la politique a pour effet d'empêcher le ministre des Transports de perdre la face ${ }^{39}$. C'est dans ces conditions que certaines ressources collectives sont mobilisées. Le ministère bénéficie de l'intervention de Jacques Delors, Premier ministre par intérim du fait du voyage de Pierre Mauroy en Autriche (François Mitterrand, lui, est en Irlande puis en Italie). Cette entrée en jeu, ajoutée à celle du président du CNPF, Yvon Gattaz, a pour effet de troubler la FNTR qui assiste à la rupture des transactions établies avec le CNPF, la CGPME et les partis politiques. "Les partis politiques ont dit qu'ils n'offraient pas leur service" et "Gattaz a appelé en nous disant que "votre place n'est pas dans la rue" (la CGPME a fait la même chose" ${ }^{40}$. C'est l'entrée en jeu de J. Delors qui permet aux uns et aux autres de mobiliser des ressources qu'ils se refusaient encore à utiliser. Jusque là, l'équipe du ministère ne pouvait (ou ne souhaitait) pas déstabiliser durablement la FNTR. J. Delors, lui, du fait de sa position institutionnelle, ne dépend pas de la stabilité des transactions établies avec les groupements de transporteurs. Il bénéficie même d'un a priori favorable car avec lui et jusque là "la négociation était bien passée" ${ }^{41}$. Or ce sont les groupements patronaux qui posent problème du fait de leurs hésitations entre un appel à la levée des barrages et des mots d'ordre de durcissement du conflit. L'entrée en jeu de J. Delors permet de mobiliser un réseau de relations dans les instances patronales pour agir sur la FNTR : brusquement celle-ci n'est plus seulement en train de perdre la face aux yeux de ses adhérents, ses dirigeants saisissent que le groupement lui-même est remis en cause. Ce qui se joue alors ce n'est plus l'impossible qualité d'industriel comme au début du XXe siècle pour les entrepreneurs de transports automobiles (de fait ils perdent cette qualité pour devenir progressivement des prestataires de services), mais le respect de leur qualité de chefs d'entreprise, de gestionnaires responsables de l'économie nationale dans un groupement autonome et 
jusque là légitime. A ces relations mobilisées par le Premier ministre par intérim s'ajoutent celles mobilisées par les membres du cabinet dans la FNTR même. Ces activations de réseaux de relations et l'émergence du noyau dur des transporteurs de marchandises contre les voyageurs ont pour effet d'imposer aux différents agents et groupements locaux un nouveau sens à leur action. Ce qu'ils font en continuant à barrer les routes (ou à encourager et à faciliter les barrages) revient désormais à contribuer à la scission de leur groupement. Cette division repose sur l'opposition entre les voyageurs et les marchandises qui tient depuis les années trente et qui semble brusquement être rompue. Dans cette période le système d'opposition de ces deux activités est mis à jour. Certains évoquent la richesse des transporteurs de voyageurs qui peuvent "partir avec la caisse de la FNTR", d'autres insistent sur la dimension sociale de ces entrepreneurs et de ces conducteurs qui ne peuvent appeler à la révolte ou barrer les routes. Cette rupture au sein même de la FNTR transforme les anticipations faites sur l'issue du conflit : les propositions ministérielles deviennent un acquis alors qu'elles étaient jusque là discutables. Sur les barrages, une autre technique de résolution des conflits est utilisée, il s'agit de la "stratégie charismatique" mise en œuvre grâce à la personnalité des leaders. En février 1984, M. Vasseur, artisan transporteur, est néanmoins reconnu par les différents agents dans les Alpes. Capable de mettre par écrit les revendications, prenant la parole pour les siens, répondant aux journalistes, cet individu représente le "petit" qui n'est pas stigmatisé. De plus, il a pendant cette période un don d'ubiquité : présent sur les barrages pour faire voter leur levée, présent ailleurs pour assister les préfets dans leur distribution de 2.000 francs en liquide aux routiers pour faciliter la levée des barrages, présent à Paris pour négocier ou à Genève pour accorder un entretien télévisée, M. Vasseur est l'individu dans lequel les différents individus en présence croient et font croire en son pouvoir ${ }^{42}$.

Le calendrier des conflits et la levée des barrages

13 Lors des trois mouvements d'envergure qu'a connus la France depuis 1984, la levée des barrages a à chaque fois surpris les agents en présence par sa rapidité et sa facilité. En 1984 principalement, les éléments climatiques laissaient présager un conflit interminable ou tout du moins une lente et difficile remise en route des véhicules: réservoirs gelés, pneus crevés ou dégonflés, véhicules incendiés, interminable succession de véhicules imbriqués les uns dans les autres au milieu de la neige, confusion presque totale sur les axes autoroutiers des Alpes et éloignement des conducteurs de leurs véhicules. Malgré ces éléments et à la surprise des membres du cabinet, les barrages se sont levés aussi vites qu'ils s'étaient constitués. La neige, le froid ou les réformes arrêtent certes les camions mais ils n'empêchent pas les conducteurs de reprendre le volant une fois l'ordre de remise en route adopté, diffusé ou intériorisé. Cette levée des barrages est due à une démobilisation et à une autolimitation du mouvement chez les entrepreneurs et conducteurs. Un des éléments importants dans l'auto-limitation de ces mouvements tient dans la mise sur pied de négociations concomitantes ou dans la semaine à venir. Tout se passe comme si les différents agents en présence (ministères, groupements et individus sur les barrages) avaient intériorisé ce calendrier des conflits qui veut que les négociations signifient la fin des hostilités et le début du travail proprement syndical - ce calendrier s'appliquant en dehors de toutes considérations portant sur les points précis de la négociation en cours. Ainsi, avant la levée des barrages, le 23 février 1984 un accord salarial est signé prévoyant l'augmentation des salaires des conducteurs de $3 \%$. Au début du mois de mars, les négociations avec le ministre aboutissent elles à la récupération progressive 
de la TVA sur le gas-oil et à l'aménagement du temps de travail du personnel de conduite. En juillet 1992, les négociations immédiatement engagées par le ministre des Transports (Jean-Louis Bianco) et le ministre du Travail (Martine Aubry) portent sur deux points juridiques qui sont au centre de la concurrence pour la définition de ce groupe social. Le "routier" est défini par équivalence avec les autres catégories salariées. Suite à ces négociations, une de ces équivalences a disparu : on ne calcule plus le temps de travail par équivalence avec les 39 heures pratiquées dans les entreprises "normales" mais en fonction des seules nécessités des entreprises de transports. Cette rupture est la première reconnaissance juridique de l'entreprise de transports au bénéfice du salarié. Sur un autre plan, les relations entre le conducteur et l'entrepreneur étaient elles aussi assurées juridiquement par les théories ordinaires de la responsabilité civile et pénale. Désormais, le conducteur n'est plus seul responsable de sa conduite, une règle spécifique s'applique dans ce secteur : le donneur d'ordre quel qu'il soit peut y être associé. Ce deuxième élément constitue la reconnaissance juridique du lien qui unit le conducteur à l'entrepreneur sans référence exogène.

Nous ne pouvons pas nous associer à l'ensemble des observateurs des mouvements sociaux et affirmer avec eux que les routiers barrent les routes. Nous ne pouvons pas plus décréter que cette technologie d'action collective soit propre à ce groupe social. Sur un siècle, il est impossible de reconnaître ce groupe grâce à cette forme ou cette forme grâce à cet utilisateur : il y a plusieurs usages de ces regroupements, plusieurs moyens de les percevoir, plusieurs groupes sociaux et même, pour les routiers, plusieurs catégories internes à un groupe qui s'en servent. Toutes ces prénotions constituées sur l'action collective nous empêchent en fait de restituer les catégories mentales (et leur évolution) qui peu à peu permettent de reconnaître un joueur (ici le groupe des "routiers") à son style (ici la violence, vulgarité, extrémisme) pour finalement l'y cantonner. Elles nous empêchent également d'apercevoir ce qui se joue dans l'imposition du sens, de l'utilité et de la causalité propre à une forme d'action collective. Elles nous empêchent enfin de relier les différentes formes d'action collective entre elles, celles qui s'apparentent à des ruptures plus ou moins violentes de l'ordre établi comme celles qui paraissent aujourd'hui les plus pacifiques. Les routiers ne sont pas violents naturellement ou volontairement mais utilisent des techniques coercitives de regroupement qui ont pour objet de retenir ceux qu'ils considèrent comme leurs semblables dans certaines situations. Les routiers ne barrent pas les routes parce que ce lieu constitue leur lieu de travail ou leur espace de liberté (leur fameux individualisme et penchant pour la solitude). En fait, les routiers ne barrent pas les routes, ils tentent de retenir un instant et d'objectiver durablement leur groupe social. 


\section{NOTES}

1. L'essentiel de cette recherche (sur archives, entretiens, dépêches et coupures de presse) porte sur les événements de février 1984. Les développements moins nombreux concernant novembre 1991 ou juillet 1992 ne proviennent que des seules coupures de la presse politique nationale.

2. Pour un point de vue radicalement opposé au notre qui prend comme unique élément de définition du groupe le Répertoire opérationnel des métiers et des emplois, cf. Lefèbvre (B.), Les dynamiques d'un espace professionnel. Le cas des routiers, Thèse pour le doctorat d'anthropologie, dir. R. Cresswell, 2 vol., Université Paris V, 1992. 3. Sur l'histoire sociale des routiers et la construction des stéréotypes nous renvoyons à notre thèse, Les routiers. Contribution à une sociologie des groupes d'intérêt, thèse pour le doctorat en science politique, dir. H. Portelli, Université Paris X Nanterre, 1993. 4. On aura reconnu ici les questions de sociologie qui ouvrent l'ouvrage que Luc Boltanski a consacré aux cadres, Les cadres. La formation d'un groupe social, Ed. de Minuit, Paris, 1982, p. 51-52).

5. Cf. Fédération nationale des moyens de transports, 1936, Compte rendu du congrès fédéral national, L'Émancipatrice, p. 93.

6. Lors de la première assemblée constitutive du Groupement syndical des transports routiers de la Seine (affilié à la FNTR), cf. La vie des transports du 3 mai 1947, nº 70, p. 5. 7. Dans le sens et selon la logique utilisés par F. Héran et M. Bozon pour ne pas redire des évidences en mettant la profession et l'origine sociale sur le papier dans leur retour sur l'analyse classique du choix du conjoint, in "La découverte du conjoint. I. Evaluation et morphologie des scènes de rencontre", Population, 6, 1987, p. 944.

8. Les barrages routiers n'ont pas été la seule technologie d'action collective utilisée par les différents groupements et catégories sociales du transport sur route. Depuis le début du XXè siècle, la grève est également utilisée. Mais la grève ne continue à être utilisée que là où les individus se pensent comme des employés ou des salariés et pour qui le véhicule n'est pas un marqueur social objectivé.

9. Dans le sens que M. Dobry donne à cet effet des conjonctures fluides sur les individus in Sociologie des crises politiques, Paris, Presses de la FNSP, 1986.

10. Les formes collectives investies par les différents agents composant ce groupe social sont le produit de l'économie psychique propre à ce groupe qui s'édifie au cours de la différenciation du secteur économique des transports au tout début du XXè siècle et que l'on observe dans la codification des règles de conduite sur route. Sur l'édification de cette économie psychique avant les années trente (notion empruntée à N. Elias et qui correspond en l'espèce à l'autolimitation produite par les regards portés sur les agents de ce groupe par les autres agents de la société) nous renvoyons à la première partie de notre thèse, op. cit.

11....

12. Note pour le Préfet, 28 juin 1913, Archives nationales, série F/7/13926.

13. Revue des agents, 1 février 1933, Archives nationales, série $F / 14 / 14974$.

14. L'Humanité, 30 mai 1909.

15....

16. L'Action, 21 octobre 1910.

$17 . .$. 
18. Note du 5 juin 1911 pour le préfet, Archives nationales, série F/7/13926.

19....

20. ...

21. Les transports automobiles, 23 octobre 1933.

22. La vie des transports, $n^{\circ} 64$, février 1947.

23. L'Officiel des transporteurs, $n^{\circ} 1153$, février 1981.

24. En juillet 1992, les accès de Paris étaient étroitement surveillés et tous les poids lourds ne possédant pas un titre de transport pour le centre ville étaient interdits d'accès.

25. Sur la critique de ce modèle particulier d'explication des comportements individuels en psychologie sociale, cf. Perrenoud (P.), "De quelques apports piagétiens à une sociologie de la pratique", Revue européenne des sciences sociales, 1976, 4, 38-39.

26. Le Monde, 13 août 1974.

27. Ibid, 12 septembre 1974.

28. En 1991, le ministre de l'Intérieur P. Marchand a saisi cette dimension des barrages tenus sur les autoroutes. Certains barrages ont très rapidement perdu toute leur efficacité quand les forces de police ont démonté les glissières de sécurité "piégeant les camionneurs non grévistes, nombre de chauffeurs en ont profité [[...

29. Sur ce point nous renvoyons à notre article, "Le sens unique. La codification des règles de conduite sur route", Politix, n¹0/11, 1990.

30. "Des routiers", Nord Eclair, 22 février 1984.

31. qui

32. Cf. Dupuy (F.), Thoenig (J.-C.), L'administration en miettes, Paris, Fayard, 1985, p. 217.

33. Sur ce point cf. Dobry (M.), Sociologie des crises politiques, op. cit.

34. Ceci n'est valable que pendant le conflit. Une fois le calme revenu et les catégories de perception re-maîtrisées, ces individus sont généralement insérés dans le champ de représentation comme troisième voie puis peu à peu contribuent à re-stabiliser la dimension binaire de l'espace social.

35. Cf. Dobry (M.), "Calcul, concurrence et gestion du sens", in Favre (P.), dir., La manifestation, Paris, Presses de la FNSP, 1990, p. 366.

36. Le Monde, 9 juillet 1992.

37. Réaction de P. Mauroy lors du comité interministériel rapportée par le directeur de cabinet de C. Fiterman.

38. Entretien avec le directeur de cabinet de C. Fiterman.

39. Interviennent successivement : B. Pons, F. Guillaume, J. Poperen, J. Chirac, le bureau exécutif du PS, F. Léotard, A. Lajoinie, C. Estier, P. Juquin.

40. Entretien avec le délégué général de la FNTR.

41. Ibid.

42. M. Vasseur traite lui-même certains stigmates en expliquant pourquoi "les gars sur les barrages étaient bourrés" (cf. le voyage de retour de M. Vasseur, Libération 27 février 1984). En juillet 1992, la même stratégie est mise en place avec "Tarzan" que les journaux télévisés montrent entrain d'acheter "un costume" pour rencontrer le Premier ministre. 


\section{RÉSUMÉS}

Les différents types de conducteurs de véhicules de poids lourds et les différents entrepreneurs de transports routiers n'ont pas toujours eu recours à la technologie d'action collective du barrage. Du début du XXè siècle à la fin des années cinquante, ces individus, "les routiers", agissent collectivement dans les grands centres urbains. A la fin des années cinquante, les rues des villes ne sont plus l'objet de ces mobilisations mais restent néanmoins un enjeu symbolique. C'est dans les années soixante que les groupements d'entrepreneurs et de conducteurs découvrent les technologies du barrage routier qui deviennent à trois reprises des événements politiques. Cette forme particulière d'action collective repose sur l'art de se regrouper de ce groupe social. Elle constitue un procédé de sélection des individus qu'il convient de retenir avec soi et repose sur les formes élémentaires de la vie collective de ce groupe social.

INDEX

Index géographique : France

Mots-clés : mobilisation, mobilité, Mouvements sociaux 